\title{
Formulas for Finding the Argument for Which a Function Has a Given Derivative
}

The problem of finding where a function $f(x)$ that is tabulated at equal intervals $h$ has a given derivative (i.e. given $f^{\prime}(x) \equiv f_{x}^{\prime} \equiv f^{\prime}\left(x_{0}+p h\right)$, to find $p$ ) arises very frequently in numerical computation, especially in the location of a maximum or minimum point when $f^{\prime}\left(x_{0}+p h\right)=0$. The usual methods for finding where the derivative of a function has a certain value are either (1) to calculate the derivatives at the tabular points and then perform inverse interpolation, (2) to estimate where the derivative has the desired value and then subtabulate the first derivative in which connection a table by SALzER ${ }^{1}$ will be found helpful, or (3) to reverse the series in $p$, obtained by differentiating any one of the direct interpolation formulas for $f\left(x_{0}+p h\right)$. The drawback in (1) is that it involves two separate steps, and the drawback in (2) is that it requires much more work than is necessary, together with a large amount of continual personal inspection. Thus method (3) which gives $p$ in terms of the tabular entries $f\left(x_{0}+m h\right)$ seems to be the most convenient, and it is recommended by GrBB, ${ }^{2}$ in the form of reversion of a series involving differences. This present note gives the coefficients of the reversed series for $p$ directly in terms of the tabular entries, so that no differences are necessary. However, if differences are already available, one might use them conveniently in the formulas given at the end which are obtained by reversing the series for the derivative of the Newton-Stirling formula using central differences $\delta_{0}{ }^{2 m}$ and mean-central differences $\mu \delta_{0}{ }^{2 m+1}$, viz.,

$$
\begin{aligned}
h f^{\prime}\left(x_{0}+p h\right) & =\left(\mu \delta_{0}-\frac{1}{6} \mu \delta_{0}^{3}+\frac{1}{30} \mu \delta_{0}{ }^{5}-\cdots\right)+p\left(\delta_{0}^{2}-\frac{1}{12} \delta_{0}^{4}+\frac{1}{90} \delta_{0}{ }^{6}-\cdots\right) \\
& +p^{2}\left(\frac{1}{2} \mu \delta_{0}^{3}-\frac{1}{8} \mu \delta_{0}^{5}+\cdots\right)+p^{3}\left(\frac{1}{6} \delta_{0}^{4}-\frac{1}{36} \delta_{0}^{6}+\cdots\right) \\
& +p^{4}\left(\frac{1}{24} \mu \delta_{0}^{5}-\cdots\right)+p^{5}\left(\frac{1}{12} \delta_{0} \delta^{6}-\cdots\right)+\cdots
\end{aligned}
$$

which, after transposition of the constant term in the right member, and division by the coefficient of $p$, assumes the form $r=p+s p^{2}+t p^{3}+u p^{4}$ $+v p^{5}+\cdots$. The formulas are for all cases where 3 through 7 points are needed in direct interpolation. These formulas are similar to the formulas for inverse interpolation by the author; ${ }^{3}$ but these combine the two operations of differentiation and inverse interpolation in a simple manner.

The function $f(x)$ requires $n$ points for direct interpolation when the interpolating polynomial which equals $f\left(x_{0}+m h\right) \equiv f\left(x_{m}\right) \equiv f_{m}$, $x_{m} \equiv x_{0}+m h, m=-[(n-1) / 2]$ to $[n / 2]$, is of the $(n-1)^{\text {th }}$ degree. Corresponding to $n=3(1) 7$, quantities $r, s, t, u$ and $v$ are defined below. (The user must note carefully that in $f_{x}{ }^{\prime} \equiv f^{\prime}(x)$, the derivative is with respect to $x$, and not $p$.)

\section{3-Point}

$r=\left(2 h f_{x}^{\prime}+f_{-1}-f_{1}\right) / D, s=t=u=v=0$,

where $D=2\left(f_{-1}-2 f_{0}+f_{1}\right)$.

\section{4-Point}

$r=\left(6 h f_{x}^{\prime}+2 f_{-1}+3 f_{0}-6 f_{1}+f_{2}\right) / D$,

$s=3\left(-f_{-1}+3 f_{0}-3 f_{1}+f_{2}\right) / D, t=u=v=0$,

where $D=6\left(f_{-1}-2 f_{0}+f_{1}\right)$. 


\section{5-Point}

$$
\begin{aligned}
r & =\left(24 h f_{x}^{\prime}+2\left(-f_{-2}+8 f_{-1}-8 f_{1}+f_{2}\right)\right) / D, \\
s & =6\left(-f_{-2}+2 f_{-1}-2 f_{1}+f_{2}\right) / D, \\
t & =4\left(f_{-2}-4 f_{-1}+6 f_{0}-4 f_{1}+f_{2}\right) / D, \\
u & =v=0,
\end{aligned}
$$

where $D=2\left(-f_{-2}+16 f_{-1}-30 f_{0}+16 f_{1}-f_{2}\right)$.

\section{6-Point}

$$
\begin{aligned}
r & =\left(120 h f_{x}{ }^{\prime}+2\left(-3 f_{-2}+30 f_{-1}+20 f_{0}-60 f_{1}+15 f_{2}-2 f_{3}\right)\right) / D, \\
s & =15\left(-f_{-2}-f_{-1}+10 f_{0}-14 f_{1}+7 f_{2}-f_{3}\right) / D \\
t & =20\left(f_{-2}-4 f_{-1}+6 f_{0}-4 f_{1}+f_{2}\right) / D \\
u & =5\left(-f_{-2}+5 f_{-1}-10 f_{0}+10 f_{1}-5 f_{2}+f_{3}\right) / D \\
v & =0
\end{aligned}
$$$$
\text { where } D=10\left(-f_{-2}+16 f_{-1}-30 f_{0}+16 f_{1}-f_{2}\right) \text {. }
$$

\section{7-Point}

$r=\left(720 h f_{x}{ }^{\prime}+12\left(f_{-3}-9 f_{-2}+45 f_{-1}-45 f_{1}+9 f_{2}-f_{3}\right)\right) / D$,

$s=45\left(f_{-3}-8 f_{-2}+13 f_{-1}-13 f_{1}+8 f_{2}-f_{3}\right) / D$,

$t=20\left(-f_{-3}+12 f_{-2}-39 f_{-1}+56 f_{0}-39 f_{1}+12 f_{2}-f_{3}\right) / D$,

$u=15\left(-f_{-3}+4 f_{-2}-5 f_{-1}+5 f_{1}-4 f_{2}+f_{3}\right) / D$,

$$
v=6\left(f_{-3}-6 f_{-2}+15 f_{-1}-20 f_{0}+15 f_{1}-6 f_{2}+f_{3}\right) / D \text {, }
$$

where $D=4\left(2 f_{-3}-27 f_{-2}+270 f_{-1}-490 f_{0}+270 f_{1}-27 f_{2}+2 f_{3}\right)$.

Then the quantity $p$ is given by

$$
\begin{aligned}
p=r-r^{2} s+r^{3}\left(2 s^{2}-t\right) & +r^{4}\left(-5 s^{3}+5 s t-u\right) \\
& +r^{5}\left(14 s^{4}-21 s^{2} t+3 t^{2}+6 s u-v\right) \\
+ & r^{6}\left(-42 s^{5}+84 s^{3} t-28 s t^{2}-28 s^{2} u+7 t u+7 s v\right)+\cdots
\end{aligned}
$$

When differences are available it is convenient to define the $r, s, t, u$ and $v$ according to the scheme below, the formula for $p$ being the same as above. However, these formulas using differences, when expressed in terms of the tabular entries $f_{m}$, are not the same as those previously given directly in terms of $f_{m}$. Thus, when differences are available, these formulas using differences might serve as a partial check on those formulas without differences, or vice versa:

\section{3-Point}

$r=\left(h f_{x}{ }^{\prime}-\mu \delta_{0}\right) / \delta_{0}{ }^{2}, s=t=u=v=0$.

$r=\left(h f_{x}^{\prime}-\mu \delta_{0}+\frac{1}{6} \mu \delta_{0}^{3}\right) / \delta_{0}{ }^{2}$,

\section{4-Point}

$s=\frac{1}{2} \mu \delta_{0}^{3} / \delta_{0}^{2}, t=u=v=0$. 
$r=\left(h f_{x}^{\prime}-\mu \delta_{0}+\frac{1}{6} \mu \delta_{0}^{3}\right) / \Delta$,

5-Point

$s=\frac{1}{2} \mu \delta_{0}^{3} / \Delta$,

$t=\frac{1}{6} \delta_{0}^{4} / \Delta, u=v=0$,

where $\Delta=\delta_{0}^{2}-\frac{1}{12} \delta_{0}^{4}$.

6-Point

$r=\left(h f_{x}{ }^{\prime}-\mu \delta_{0}+\frac{1}{6} \mu \delta_{0}{ }^{3}-\frac{1}{30} \mu \delta_{0}{ }^{5}\right) / \Delta$,

$s=\left(\frac{1}{2} \mu \delta_{0}^{3}-\frac{1}{8} \mu \delta_{0}^{5}\right) / \Delta$,

$t=\frac{1}{6} \delta_{0}^{4} / \Delta$,

$u=\frac{1}{24} \mu \delta_{0}{ }^{5} / \Delta, v=0$,

where $\Delta=\delta_{0}^{2}-\frac{1}{12} \delta_{0}^{4}$.

\section{7-Point}

$r=\left(h f_{x}^{\prime}-\mu \delta_{0}+\frac{1}{6} \mu \delta_{0}^{3}-\frac{1}{30} \mu \delta_{0}^{5}\right) / \Delta$,

$s=\left(\frac{1}{2} \mu \delta_{0}^{3}-\frac{1}{8} \mu \delta_{0}^{5}\right) / \Delta$,

$t=\left(\frac{1}{6} \delta_{0}^{4}-\frac{1}{36} \delta_{0}^{6}\right) / \Delta$,

$u=\frac{1}{24} \mu \delta_{0}^{5} / \Delta$,

$v=\frac{1}{12} \sigma_{0} \delta_{0} / \Delta$,

where $\Delta=\delta_{0}^{2}-\frac{1}{12} \delta_{0}^{4}+\frac{1}{80} \delta_{0}^{6}$.

H. E. SAlzer

National Bureau of Standards

Washington 25, D. C.

This work was sponsored in part by the Office of Air Research.

${ }^{1} \mathrm{H}$. E. SALZER, "Table of coefficients for obtaining the first derivative without differences," NBS, Applied Mathematics Series No. 2, 1948.

${ }^{2} \mathrm{D}$. GIBB, $A$ Course in Interpolation and Numerical Integration for the Mathematical Laboratory. London, Bell, 1915.

${ }^{3} \mathrm{H}$. E. SALZER, "A new formula for inverse interpolation," Amer. Math. Soc., Bull., v. 50,1944 , p. $513-516$

\section{RECENT MATHEMATICAL TABLES}

915[F].-K. FRÜCHTL, "Statistische Untersuchung über die Verteilung von Primzahl-Zwillingen," Öster. Akad. Wiss., math.-nat. Kl., Anz., 1950, p. 226-232.

Information on the distribution of twin primes $(p, p+2)$ and quadruplets $(p, p+2, p+6, p+8)$ is given for the first 1020000 natural numbers. The information is based on the old table of Chernac. ${ }^{1}$ The main table gives the number of prime pairs in each of the 1020 chileads $1000 n<p<1000(n+1)$ for $n=0(1) 1019$. There is no chilead devoid of prime pairs and only three $(n=688,851,927)$ with but a single prime pair. The rows of this table are added to give the number of prime pairs in each of the 102 myriads $10000 n<p<10000(n+1)$ for $n=0$ (1)101. These frequencies are, in turn, added by tens to give a 10 entry table for each interval of 100000 . The grand total gives 8168 prime pairs $<10^{6}$. 